\title{
Cryptanalysis of a Biometric-based Multi-Server Authentication Scheme
}

\author{
Tao Wan ${ }^{1,}$, Nan Jiang ${ }^{2}$, Jianfeng Ma ${ }^{1}$, Lin Yang ${ }^{3}$ \\ ${ }^{1}$ School of Computer Science and technology, Xidian University, \\ $X i$ 'an 710071, China \\ ${ }^{2}$ School of Information Engineer, East China Jiaotong University, \\ Nanchang 330013, China \\ ${ }^{3}$ The Research Institute, China Electronic Equipment and Systems Engineering \\ Corporation, Beijing 100039, China \\ 1wantao217@163.com, ${ }^{2}$ jiangnan1018@gmail.com
}

\begin{abstract}
Authentication and key agreement protocol becomes an important security issue for multi-server architecture. Combining biometrics with password enhances the level of security. Recently, Baruah et al. analyzed that Mishra et al.'s protocol has several drawbacks and proposed an improved biometric based multi-server authentication scheme. They claimed that their scheme satisfies all the required security attributes for a secure authentication. In this paper, we indicate that their scheme is not secure against key reveal attack, replay attack, and smart card forgery attack. Any registered user can retrieve the session key or launch the replay attack by eavesdropping on the communication channel. In addition, registered user can forge smart card when colluding with registered server.
\end{abstract}

Keywords: Authentication, Multi-server, Biometric, Smart card

\section{Introduction}

With the rapid development of Internet service, remote user authentication scheme becomes an important issue for practical applications. More and more network architectures are used in multi-server environments. However, it is extremely hard for a user to remember these numerous different identities and passwords when he/she uses the single-server authentication protocol to login and access different remote service providing servers. In order to resolve this problem, many multi-server authentication and key agreement schemes have been proposed.

In 2001, Li et al. [1] first proposed the concept of multi-server authentication protocol. But their scheme need large memory and high computational cost. In 2004, Tsaur et al. [2] designed a multi-server authentication scheme based on the RSA cryptosystem and Lagrange interpolating polynomial. But their scheme is subject to high communication and computation costs. In 2008, Tsai [3] proposed a multi-server authentication scheme based on the nonce and one-way hash function. ${ }^{1}$ However, his scheme was found susceptible to the server spoofing and the impersonation attacks [4]. In 2008, Lee et al. [5] proposed an efficient remote authenticated key agreement scheme for multi-server by adopting hash function and exclusive-OR. Nevertheless, Chang et al. [6] indicated that their scheme is vulnerable to the forgery attack. In 2009, Liao and Wang [7] designed a dynamic identity based remote user authentication protocol for multi-server environment to achieve user's anonymity. However, this scheme was found to be vulnerable to insider attack, masquerade attack, server spoofing attack, and registration center spoofing attack

Tao Wan is the corresponding author. 
by Hsiang and Shih [8]. Wan et al. [9] analyzed that two dynamic ID based remote user authentication schemes for multi-server environment proposed by Lee et al. [10] and $\mathrm{Li}$ et al. [11] were susceptible to stolen smart card attack, leak-of-verifier attack and so on.

More recently, many researches have combined user's biometrics (e.g., fingerprints, irises, and hand geometry) with password and smart card to design remote user authentication scheme to enhance the level of security. The main feature of using biometric is its uniqueness. Yang et al. [12] and Yoon et al. [13] proposed biometric based multi-server authentication schemes, but they did not consider the user anonymity. Moreover, Yang et al.'s scheme need high computational cost, and Yoon et al.'s scheme was found by He [14] to be vulnerable to insider attack, masquerade attack, and stolen smart card attack.

Recently, Chuang et al. [15] introduced an anonymous biometric based multi-server authentication scheme. But Mishra et al. [16] demonstrated their scheme was vulnerable to stolen smart card attack, impersonation attack and server spoofing attack, and proposed an improved multi-server authentication scheme. However, Baruah et al. [17] found that their scheme still cannot withstand stolen smart card attack and impersonation attack, and then proposed an enhanced authentication scheme. They declaimed their scheme satisfies all the required security attributes. Unfortunately, we identify that Baruah et al.'s scheme is susceptible to key reveal attack, replay attack and smart card forgery attack.

The remainder of this manuscript is organized as follows. We review the biometric based multi-server authentication protocol proposed by Baruah et al. in Section 2. We analyze the security flaws of Baruah et al.'s protocol in Section 3. We conclude this paper in Section 4.

\section{Review of Baruah et al.'s Scheme}

Here we will review Baruah et al.'s biometric based multi-server authentication scheme. The notations used throughout this paper are summarized in Table 1.

Table 1. Notations used in the paper

\begin{tabular}{|l|l|}
\hline Symbols & \multicolumn{1}{|c|}{ Their meaning } \\
\hline$R C$ & the registration center \\
$U_{i}$ & the $i_{\text {th }}$ user \\
$I D_{i}$ & the identity of $U_{i}$ \\
$P W_{i}$ & the password of $U_{i}$ \\
$B I O_{i}$ & the biometric of $U_{i}$ \\
$P S K$ & Pre-shared key \\
$S_{j}$ & the $j_{\text {th }}$ server \\
$S I D_{j}$ & the identity of $S_{j}$ \\
$x$ & the master secret key of $R C$ \\
$h(\cdot)$ & a secure one way hash function \\
$\oplus$ & Exclusive-OR operation \\
$\|$ & string concatenation operation \\
\hline
\end{tabular}

Their scheme involves three participants, the user $U_{i}$, the server $S_{j}$ and the registration center $R C$. Their scheme can be divided into five phases: server registration phase, user registration phase, login phase, authentication phase and password change phase. We show the login and authentication phases in Figure 1. More details are provided in the following.

\subsection{Server Registration Phase}

An application server $S_{j}$ sends a registration request along with its identity $S I D_{j}$ to the registration center $R C$, if he wishes to become a registered server. Then the registration center $R C$ chooses the master key $x$ and the pre-shared key PSK to compute 
$h\left(S I D_{j} \| h(P S K)\right)$ and $h(P S K \| x)$, then sends them to the application server $S_{j}$ using the Internet Key Exchange Protocol (IKEv2) [18].

\subsection{User Registration Phase}

When a new user $U_{i}$ wishes to access any services provided by the registered servers, he must first register himself. This registration phase consists of the following steps:

Step R1: The user $U_{i}$ freely chooses his identity $I D_{i}$, password $P W_{i}$ and personal biometric $B I O_{i}$, and computes $R_{i}=h\left(P W_{i} \| B I O_{i}\right)$. Then $U_{i}$ sends $I D_{i}$ and $R_{i}$ to $R C$ over a secure channel.

Step R2: $R C$ computes $A_{i}=h\left(I D_{i} \| x\right), B_{i}=h(P S K \| x) \oplus A_{i}, C_{i}=h\left(R_{i} \| I D_{i}\right) \oplus h\left(A_{i}\right), D_{i}=$ $h(P S K) \oplus h\left(I D_{i}\right), E_{i}=R_{i} \oplus I D_{i}$, and securely issues the smart card containing $\left\{B_{i}, C_{i}, D_{i}, E_{i}\right.$, $h(\cdot)\}$ to the user $U_{i}$.

\subsection{Login Phase}

Step L1: $U_{i}$ inserts his smart card and inputs his identity $I D_{i}$, password $P W_{i}$ and personal biometric $B I O_{i}$. The smart card computes $R_{i}=h\left(P W_{i} \| B I O_{i}\right)$, and checks whether the entered identity $I D_{i}$ is equal to $E_{i} \oplus R_{i}$. If it holds, the legitimacy of $U_{i}$ can be assured.

Step L2: The smart card computes $h(P S K)=h\left(I D_{i}\right) \oplus D_{i}$ and $h\left(A_{i}\right)=C_{i} \oplus h\left(R_{i} \| I D_{i}\right)$, then generates a nonce $N_{i}$, and computes $M_{1}=h\left(S I D_{j} \| h(P S K)\right) \oplus h\left(I D_{i} \| N_{i}\right), M_{2}=N_{i} \oplus h\left(A_{i}\right), V_{1}=$ $h\left(N_{i} \oplus B_{i}\right)$.

Afterwards, the smart card sends the login request massage $\left\{B_{i}, M_{1}, M_{2}, V_{1}\right\}$ to the server $S_{j}$ via a public channel.

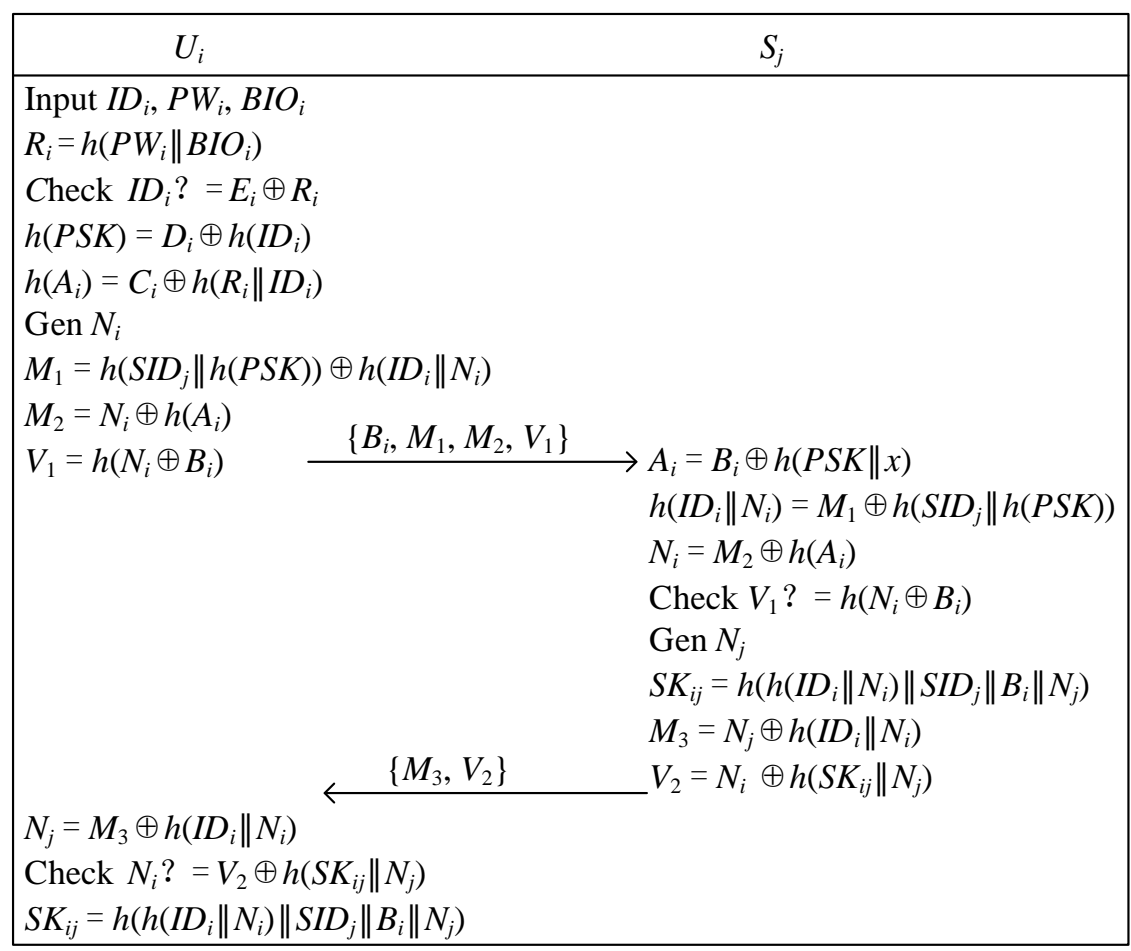

Figure 1. Login and Authentication Phase of Baruah et al.'s Scheme

\subsection{Authentication Phase}

Step V1: Once $S_{j}$ receives the login request massage $\left\{B_{i}, M_{1}, M_{2}, V_{1}\right\}, S_{j}$ computes $A_{i}=$ $B_{i} \oplus h(P S K \| x), h\left(I D_{i} \| N_{i}\right)=M_{1} \oplus h\left(\operatorname{SID}_{j} \| h(P S K)\right), N_{i}=M_{2} \oplus h\left(A_{i}\right)$. 
Step V2: $S_{j}$ computes $h\left(N_{i} \oplus B_{i}\right)$ and checks it with $V_{1}$. If they are equivalent, $S_{j}$ accepts the login request. Then $S_{j}$ generates a nonce $N_{j}$ to compute $S K_{i j}=h\left(h\left(I D_{i} \| N_{i}\right)\left\|S I D_{j}\right\| B_{i} \| N_{j}\right)$, $M_{3}=N_{j} \oplus h\left(I D_{i} \| N_{i}\right), V_{2}=N_{i} \oplus h\left(S K_{j i} \| N_{j}\right)$. Finally, $S_{j}$ sends the message $\left\{M_{3}, V_{2}\right\}$ to $U_{i}$.

Step V3: Upon receiving the message $\left\{M_{3}, V_{2}\right\}, U_{i}$ computes $N_{j}=M_{3} \oplus h\left(I D_{i} \| N_{i}\right), S K_{i j}=$ $h\left(h\left(I D_{i} \| N_{i}\right)\left\|S I D_{j}\right\| B_{i} \| N_{j}\right)$, and compares $N_{i}$ with $V_{2} \oplus h\left(S K_{j i} \| N_{j}\right)$. If they are equivalent, $U_{i}$ authenticates $S_{j}$.

After the mutual authentication, $U_{i}$ and $S_{j}$ can use the current session key $S K_{i j}=$ $h\left(h\left(I D_{i} \| N_{i}\right)\left\|S I D_{j}\right\| B_{i} \| N_{j}\right)$ for securing communication.

\subsection{Password Change Phase}

This phase is invoked whenever $U_{i}$ wants to change his password $P W_{i}$ to a new password $P W_{i}^{*}$.

Step P1: $U_{i}$ inserts his smart card and inputs his identity $I D_{i}$, password $P W_{i}$ and personal biometric $B I O_{i}$.

Step P2: The smart card computes $R_{i}=h\left(P W_{i} \| B I O_{i}\right)$, and checks whether the entered identity $I D_{i}$ is equal to $E_{i} \oplus R_{i}$. If it holds, $U_{i}$ chooses a new password $P W_{i}^{*}$ to compute $R_{i}^{*}=h\left(P W_{i}^{*} \| B I O_{i}\right), C_{i}^{*}=h\left(R_{i}^{*} \| I D_{i}\right) \oplus h\left(R_{i} \| I D_{i}\right) \oplus C_{i}$, and $E_{i}^{*}=E_{i} \oplus R_{i} \oplus R_{i}^{*}$.

Step P3: The smart card stores $C_{i}^{*}, E_{i}^{*}$ to replace $C_{i}, E_{i}$ respectively.

\section{Security Analysis of Baruah et al.'s scheme}

In Baruah et al.'s scheme, every servers has different secret information $h\left(\operatorname{SID}_{j} \|\right.$ $P S K)$, so their scheme can successful thwart server masquerading attack. Unfortunately, we find that their scheme still has many vulnerabilities. Any registered but malicious user can not only derive the session key between any user and server by eavesdropping their communication information in public channel, but also masquerade as the user to $\log$ into the server. In addition, when a registered but malicious user colludes with a server, they can successful log into any server by forging smart card.

\subsection{Key Reveal Attack}

From the login phase of Baruah et al.'s scheme, we find that each registered user knows $h(P S K)$. If a legal but malicious user $U_{z}$ can eavesdrop the valid login request message $\left\{B_{i}, M_{1}, M_{2}, V_{1}\right\}$ of $U_{i}$ and the authentication message $\left\{M_{3}, V_{2}\right\}$ of $S_{j}$ on the public channel, he can compute $h\left(I D_{i} \| N_{i}\right)=M_{1} \oplus h\left(S I D_{j} \| h(P S K)\right), N_{j}=M_{3} \oplus$ $h\left(I D_{i} \| N_{i}\right)$, and $S K_{i j}=h\left(h\left(I D_{i} \| N_{i}\right)\left\|S I D_{j}\right\| B_{i} \| N_{j}\right)$. Then $U_{z}$ easily derive the current session key $S K_{i j}$ shared between $U_{i}$ and $S_{j}$. After that, the attacker $U_{z}$ can decrypt all encrypted information between $U_{i}$ and $S_{j}$.

\subsection{Replay Attack}

From the above analysis, we know that any legal user $U_{z}$ can retrieve $h\left(I D_{i} \| N_{i}\right)$ by eavesdropping the login request message $\left\{B_{i}, M_{1}, M_{2}, V_{1}\right\}$ of $U_{i}$. Then $U_{z}$ can also replay the message $\left\{B_{i}, M_{1}, M_{2}, V_{1}\right\}$ to $S_{j}$. This verification holds, since the messages has not been modified. Then $S_{j}$ selects a nonce $N_{j}{ }^{\prime}$, generates the session key as $S K_{i j}{ }^{\prime}=h\left(h\left(I D_{i} \| N_{i}\right)\left\|S I D_{j}\right\| B_{i} \| N_{j}{ }^{\prime}\right)$, and computes the authentication messages $M_{3}^{\prime}=N_{j}^{\prime} \oplus h\left(I D_{i} \| N_{i}\right), V_{2}^{\prime}=N_{i} \oplus h\left(S K_{i j}^{\prime} \| N_{j}^{\prime}\right)$. Using the received authentication message $\left\{M_{3}{ }^{\prime}, V_{2}{ }^{\prime}\right\}, U_{z}$ can compute $N_{j}{ }^{\prime}=M_{3}{ }^{\prime} \oplus h\left(I D_{i} \| N_{i}\right)$ and $S K_{i j}{ }^{\prime}=h\left(h\left(I D_{i} \| N_{i}\right)\left\|S I D_{j}\right\| B_{i} \| N_{j}^{\prime}\right)$.

At last, the legal but malicious user $U_{z}$ successful masquerade as $U_{i}$ to $\log$ into the server $S_{j}$. 


\subsection{Smart card Forgery Attack}

As shown in Baruah et al.'s scheme, any registered server has the same

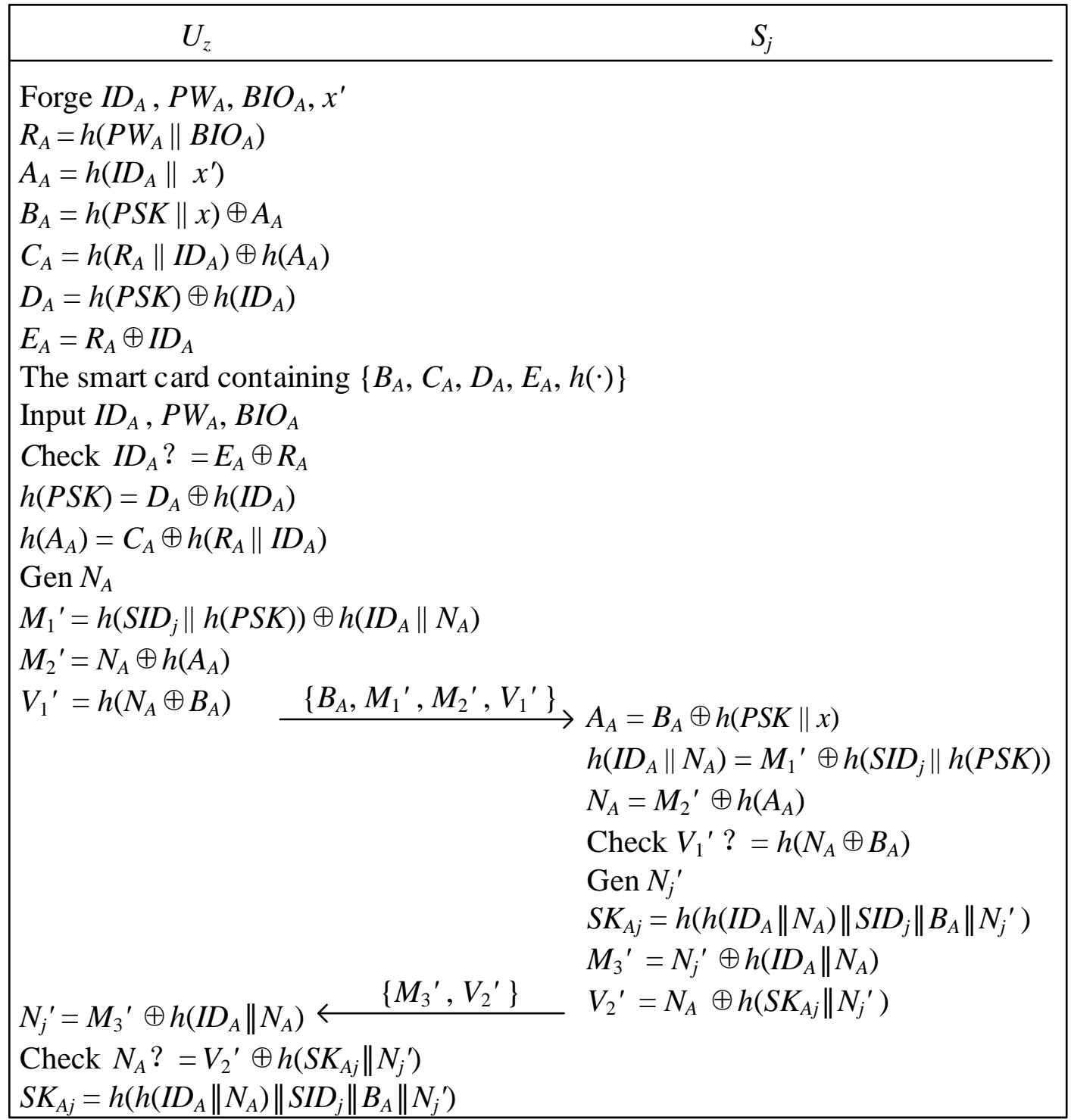

Figure 2. Smart Card Forgery Attack on Baruah et al.'s Scheme

Information $h(P S K \| x)$ and any registered user can derive the information $h(P S K)$. Under the condition that the registered but malicious user $U_{z}$ colludes with the registered but malicious $S_{k}$, they can forge a smart card to $\log$ in to any registered server (e.g., $S_{j}$ ) without knowing the personal biometric as show in Figure 2. The procedure is as follow:

- forge a new identity $I D_{A}$, password $P W_{A}$ and personal biometric $B I O_{A}$, and forge a master key $x$ '.

- compute $R_{A}=h\left(P W_{A} \| B I O_{A}\right), A_{A}=h\left(I D_{A} \| x^{\prime}\right), B_{A}=h(P S K \| x) \oplus A_{A}, C_{A}=h\left(R_{A} \|\right.$ $\left.I D_{A}\right) \oplus h\left(A_{A}\right), D_{A}=h(P S K) \oplus h\left(I D_{A}\right), E_{A}=R_{A} \oplus I D_{A}$, then the forged smart card containing $\left\{B_{A}, C_{A}, D_{A}, E_{A}, h(\cdot)\right\}$.

- insert the forged smart card and input identity $I D_{A}$, password $P W_{A}$ and personal biometric $B I O_{A}$. Obviously the legitimacy of user can be assured.

- $\quad$ the forged smart card computes $h(P S K)=h\left(I D_{A}\right) \oplus D_{A}$ and $h\left(A_{A}\right)=C_{A} \oplus h\left(R_{A} \| I D_{A}\right)$, then generates a nonce $N_{A}$, and computes $M_{1}{ }^{\prime}=h\left(S I D_{j} \| h(P S K)\right) \oplus h\left(I D_{A} \| N_{A}\right), M_{2}$ ' 
$=N_{A} \oplus h\left(A_{A}\right), V_{1}{ }^{\prime}=h\left(N_{A} \oplus B_{A}\right)$. Then, the forged smart card sends the login request massage $\left\{B_{A}, M_{1}{ }^{\prime}, M_{2}{ }^{\prime}, V_{1}{ }^{\prime}\right\}$ to the server $S_{j}$ via a public channel.

Upon receiving the message $\left\{B_{A}, M_{1}{ }^{\prime}, M_{2}{ }^{\prime}, V_{1}{ }^{\prime}\right\}, S_{j}$ computes $A_{A}=h(P S K \| x) \oplus B_{A}$, $h\left(I D_{A} \| N_{A}\right)=M_{1}{ }^{\prime} \oplus h\left(S_{I} D_{j} \| h(P S K)\right), N_{A}=M_{2}{ }^{\prime} \oplus h\left(A_{A}\right)$, and compares $h\left(N_{A} \oplus B_{A}\right)$ with $V_{1}$ '. Because they are equivalent, $S_{j}$ will accept the login request.

Then $S_{j}$ generates a nonce $N_{j}$ ' to compute $S K_{A j}=h\left(h\left(I D_{A} \| N_{A}\right)\left\|S I D_{j}\right\| B_{A} \| N_{j}{ }^{\prime}\right), M_{3}{ }^{\prime}=$ $N_{j}^{\prime} \oplus h\left(I D_{A} \| N_{A}\right), V_{2}{ }^{\prime}=N_{A} \oplus h\left(S K_{A j} \| N_{j}^{\prime}\right)$. Finally, $S_{j}$ sends the message $\left\{M_{3}{ }^{\prime}, V_{2}{ }^{\prime}\right\}$ to $U_{z}$.

When receiving the message $\left\{M_{3}{ }^{\prime}, V_{2}{ }^{\prime}\right\}, U_{z}$ computes $N_{j}^{\prime}=M_{3}^{\prime} \oplus h\left(I D_{A} \| N_{A}\right), S K_{A j}=$ $h\left(h\left(I D_{A} \| N_{A}\right)\left\|S I D_{j}\right\| B_{A} \| N_{j}^{\prime}\right)$, and compares $N_{A}$ with $V_{2}{ }^{\prime} \oplus h\left(S K_{A j} \| N_{j}^{\prime}\right)$.

At last, the attacker $U_{z}$ logs in to the server $S_{j}$ using the forged smart card. Therefore, Baruah et al.'s scheme cannot withstand smart card forgery attack.

\section{Conclusions}

Secure communication without repeating registration and biometrics based authentication are two important issues over multi-server environments. In this paper, we analyzed Baruah et al.'s biometric-based multi-server authentication scheme. Our analysis reveals its inherent security vulnerabilities, i.e., key reveal attack, replay attack and smart card forgery attack. In the future, we plan to propose an improved version of their scheme and these security weaknesses should be considered for multi-server networks.

\section{Acknowledgments}

The author would like to thank Prof. Jianfeng Ma and the anonymous referees for their constructive comments. This research was supported by the Key Program of NSFCGuangdong Union Foundation under Grant No.U1135002.

\section{References}

[1] L.H. Li, I.C. Lin, M.S. Hwang, "A remote password authentication scheme for multi-server architecture using neural networks", Neural Networks, IEEE Transactions on, vol. 12, no. 6, (2001), pp. 1498-1504.

[2] W.J. Tsaur, C.C. Wu, W.B. Lee, "A smart card-based remote scheme for password authentication in multi-server Internet services”, Computer Standard \& Interfaces, vol. 27, no. 1, (2004), pp. 39-51.

[3] J.L. Tsai, "Efficient multi-server authentication scheme based on one-way hash function without verification table", Computers \& Security, vol. 27, no. 3, (2008), pp. 115-121.

[4] R.C. Wang, W.S. Juang, C.L. Lei, "User authentication scheme with privacy-preservation for multiserver environment", Communications Letters, IEEE, vol. 13, no. 2, (2009), pp. 157-159.

[5] J.H. Lee, D.H. Lee, "Efficient and secure remote authenticated key agreement scheme for multi-server using mobile equipment", Consumer Electronics, 2008. ICCE 2008. Digest of Technical Papers. International Conference on. IEEE, (2008) January 1-2.

[6] C.C. Chang, T.F. Cheng, "A robust and efficient smart card based remote login mechanism for multiserver architecture”, Int. J. Innov. Comput. Inf. Control, vol. 7, no. 8, (2011), pp. 4589-4602.

[7] Y.P. Liao, S.S. Wang, "A secure dynamic ID based remote user authentication scheme for multi-server environment”, Computer Standards \& Interfaces, vol. 31, no. 1, (2009), pp. 24-29.

[8] H.C. Hsiang, W.K. Shih, "Improvement of the secure dynamic ID based remote user authentication scheme for multi-server environment”, Computer Standards \& Interfaces, vol. 31, no. 6, (2009), pp. $1118-1123$.

[9] T. Wan, N. Jiang, J.F. Ma, "Cryptanalysis of two dynamic identity based authentication schemes for multi-server architecture", Communications, China, vol. 11, no.11, (2014), pp. 125-134.

[10] C.C. Lee, T.H. Lin, R.X Chang, "A secure dynamic ID based remote user authentication scheme for multi-server environment using smart cards", Expert Systems with Applications, vol. 38, no.11, (2011), pp. 13863-13870.

[11] X. Li, J. Ma, W. Wang, Y. Xiong, J. Zhang, “A novel smart card and dynamic ID based remote user authentication scheme for multi-server environments", Mathematical and Computer Modelling, vol. 58, no.1, (2013), pp. 85-95.

[12] D. Yang, B. Yang, "A biometric password-based multi-server authentication scheme with smart card", Computer Design and Applications (ICCDA), 2010 International Conference on. IEEE, vol. 5, (2010), pp. $554-559$. 
[13] E.J. Yoon, K.Y. Yoo, "Robust biometrics-based multi-server authentication with key agreement scheme for smart cards on elliptic curve cryptosystem", The Journal of Supercomputing, vol. 63, no.1, (2013), pp. 235-255.

[14] D. He, "Security flaws in a biometrics-based multi-server authentication with key agreement scheme", IACR Cryptology ePrint Archive, (2011), pp. 365.

[15] M.C. Chuang, M. C. Chen, "An anonymous multi-server authenticated key agreement scheme based on trust computing using smart cards and biometrics", Expert Systems with Applications, vol. 41, no.4, (2014), pp. 1411-1418.

[16] D. Mishra, A.K. Das, S. Mukhopadhyay, "A secure user anonymity-preserving biometric-based multiserver authenticated key agreement scheme using smart cards", Expert Systems with Applications, vol. 41, no.18, (2014), pp. 8129-8143.

[17] K.C. Baruah, S. Banerjee, M.P. Dutta, C.T. Bhunia, "An Improved Biometric-based Multi-server Authentication Scheme Using Smart Card", International Journal of Security and Its Applications, vol. 9, no.1, (2015), pp. 397-408.

[18] C. Kaufman, Internet key exchange (IKEv2) protocol, (2005)
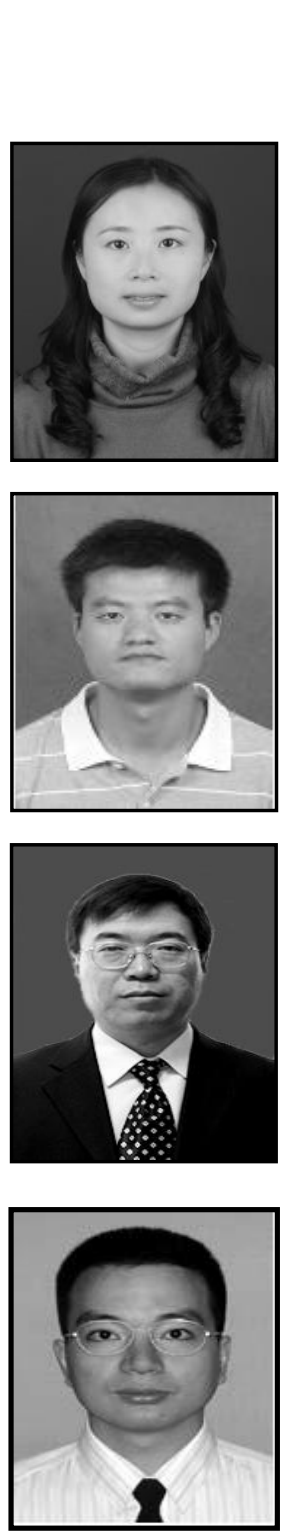

\section{Authors}

Tao Wan, She received the B.S. degree in Mathematics from Hunan University, Changsha, China in 1997, and obtained the M.S. degree in Computer Science from Xidian University, China 2003. She is currently a Ph.D. candidate at Xidian University, Xi'an, Shaanxi, China. Her research interests include cryptography, network and information security, e-commerce security technology.

Nan Jiang, He received his Ph.D. degree in Computer Application Technology from Nanjing University of Aeronautics and Astronautics, Nanjing, China, in 2008. Now he is an associate professor at East China Jiaotong University. From 2013 to 2014 he is a research scholar in Complex Networks and Security Research Lab at Virginia Tech. His research interests lie in wireless sensor networks, wireless protocol and architecture, distributed computing and complex network theory.

Jianfeng Ma, He received his M.S. and Ph.D. degrees in Computer Software and Communications Engineering from Xidian University, China in 1988 and 1995, respectively. He is an IEEE member and a senior member of CIE. Now he is a Professor and Ph.D. supervisor at Xidian University. His current research interests include distributed systems, wireless and mobile computing systems, computer networks, and information and network security.

Lin Yang, He received the B.E., M.E. and Ph.D. degrees from National University of Defense Technology of China in 1993, 1996 and 1998 respectively. He is a researcher in the Research Institute, China Electronic Equipment and Systems and doctorial supervisor of Xidian University and National University of Defense Technology. His research interests include system security and network security. 
International Journal of Security and Its Applications

Vol. 10, No. 2 (2016) 